\title{
The role of choice in vigilance performance
}

\author{
WILLIAM N. DEMBER, TRACI L. GALINSKY, and JOEL S. WARM \\ University of Cincinnati, Cincinnati, Ohio
}

\begin{abstract}
This study tested the hypothesis that affording subjects the illusion of choice with respect to the experimental condition in which they were to participate would lead to increased commitment and hence persistence in a vigilance task. Half of the subjects were offered the opportunity to select a "hard" or "easy" version of the task prior to the start of the vigil. The remaining subjects were not given that opportunity. Actually, assignment to the difficult (low signal salience) and easy (high signal salience) conditions was determined at random. In agreement with the hypothesis in question, the detection scores of the choice subjects remained more stable over the course of a 40-min vigil than did those of the controls. The results highlight the importance of intrinsic motivation in vigilance performance.
\end{abstract}

Vigilance, or sustained attention, tasks require subjects to remain alert and to detect specified critical signals over prolonged periods of time. It is typical in such tasks for critical signals to be perceptually inconspicuous events that occur with low probability (typically from about .02 to .05) in a temporally unpredictable manner within a repetitive cascade of nonsignal stimuli. It is also characteristic of such tasks that subjects' responses have little or no effect on critical signal occurrences (Warm, 1984). Hence, vigilance tasks represent situations in which subjects are confronted with tedium, uncertainty, and lack of control and in which they report considerable stress (Galinsky, 1991; Hovanitz, Chin, \& Warm, 1989; Warm, Dember, \& Parasuraman, 1991).

Given the nature of vigilance tasks, one might anticipate that motivation would play a major role in the degree to which subjects are willing to apply themselves fully to their assignment. Indeed, Smith (1966) has suggested that the vigilance decrement-the decline in efficiency over time, which is a recurrent finding in vigilance experiments (Davies \& Parasuraman, 1982)-comes from merging the scores of conscientious subjects with those of "periodic participators," individuals who lack the commitment and dedication to maintain attention to the task to the limit of their ability. The importance of commitment in laboratory studies of vigilance has also been stressed by Nachreiner (1977).

Several lines of evidence provide empirical support for the importance of motivation in vigilance experiments. For example, furnishing subjects knowledge of results about performance efficiency is a major means of enhancing

Portions of this paper were presented at the meeting of the American Psychological Association, San Francisco, 1991. We thank Michele Binder and Joel Diemler for preliminary work on this study and Amy Thatcher and Greg Handleton for collecting the data reported herein. T. L. Galinsky is now at the National Institute for Occupational Safety and Health Taft Laboratory, Cincinnati, $\mathrm{OH}$. Correspondence regarding this manuscript should be addressed to W. N. Dember, Department of Psychology, University of Cincinnati, Cincinnati, $\mathrm{OH} \mathrm{45221-0376.}$ the quality of their vigilant behavior. In some cases, however, it does not matter whether the knowledge that is supplied is veridical or nonveridical; merely the opportunity to receive evaluative information enhances the subjects' performance (Loeb \& Schmidt, 1963; Warm, Epps, \& Ferguson, 1974). Other experimenters have reported that vigilance performance can be enhanced by financial incentives (Bevan \& Turner, 1965; Sipowicz, Ware, \& Baker, 1962) or by academic incentives (Halcomb \& Blackwell, 1969). In addition, Putz (1975) has shown that vigilance performance is augmented when subjects know they are under surveillance either through having an experimenter with them in the testing room or through closedcircuit TV or one-way window modes of supervision.

The present experiment addresses an additional motivational variable, choice, or the option to select among different opportunities for action. The motivational effects of having a sense of choice have been demonstrated in a variety of social psychological settings ranging from the production and reduction of cognitive dissonance to health maintenance (Brehm \& Cohen, 1962; Rodin \& Langer, 1977). As far as we know, choice has hitherto not been manipulated in a vigilance task. However, an observation by Brickman (1987) led us to believe that it might also be an effective motivational factor in this type of task. According to Brickman, people often evidence greater commitment to difficult than to easy tasks, and hence superior performance, but only if they have chosen to undertake such tasks and not if they perceive the tasks to be imposed on them. In laboratory vigilance experiments, the canons of good experimental design call for subjects to be randomly assigned to conditions, and once they agree to serve in the experiment, they experience little choice over what transpires. In terms of Smith's (1966) analysis, that lack of perceived choice may contribute to a relatively weak commitment to the experiment and to performing as well as possible, especially as time goes on. Hence, we asked in this study whether giving subjects the illusion of choice would lead to increased commitment and persistence in the face of a lengthy, tedious, and in- 
deed stressful task. If so, we should observe in such subjects a moderation of the vigilance decrement. This investigation tested that hypothesis.

\section{METHOD}

Subjects

Eighty students, $\mathbf{4 0}$ men and $\mathbf{4 0}$ women, from introductory psychology classes at the University of Cincinnati served as subjects. All of the students had normal or corrected-to-normal vision and participated for course credit.

\section{Procedure}

A male and a female experimenter each tested half of the subjects, who were assigned at random to either a choice or a no-choice condition. The latter received standard instructions about the vigilance task:

\begin{abstract}
In this experiment, a bar of light will be flashed repetitively on the computer screen. Normally, the bar will be flashed for a normal duration. You are not required to respond to those flashes. Occasionally, however, flashes of a shorter duration will occur. These shorter flashes are referred to as critical signals. Your job will be to detect those signals whenever they appear, by pressing the computer spacebar.
\end{abstract}

Subjects in the choice condition were told in addition:

There are two possible tasks in this experiment, either a difficult or a relatively easy task. In the difficult task, the critical signals are only slightly shorter in duration than the standard bar. In the easy task, the critical signals are even shorter than in the difficult task. In other words, in the easy task the discrepancy between the standard bar and the critical signals is more pronounced. In behavioral research, subjects are not usually permitted to choose the condition they get. However, in this experiment, you may choose whether to get the difficult or the easy task. So, please tell me, which one do you prefer, difficult or easy?

In reality, subjects were randomly assigned to a difficulty condition regardless of choice. For all subjects, the duration of the standard bar (neutral events) was $247.5 \mathrm{msec}$. In the easy, or high-salience, condition, the duration of critical signals was $125 \mathrm{msec}$, a reduction of $50 \%$ from normal, whereas in the difficult, or low-salience, condition, the critical signal duration was $200 \mathrm{msec}$, a reduction of only $20 \%$ from normal. Equal numbers of male and female subjects were assigned to each of the four orthogonal cells of the design (choice/easy, choice/hard, no-choice/easy, no-choice/hard).

All subjects participated in a 50-min vigil divided into five consecutive 10-min periods. They monitored the duration of a vertically oriented $2 \times 9-\mathrm{mm}$ white bar, which appeared against a gray background in the center of a video terminal. The luminance of the bar was $21.30 \mathrm{~cd} / \mathrm{m}^{2}$, whereas that of the surround in which it appeared was $0.43 \mathrm{~cd} / \mathrm{m}^{2}$. Flashes of the bar occurred once every $1.5 \mathrm{sec}$ (event rate $=40$ events/minute) in all experimental conditions. In each condition, 10 critical signals were presented per period of watch (signal probability = $.025)$. Subjects were provided with a 6-min practice session prior to the start of the main vigil.

Subjects were tested individually in $1.95 \times 1.90 \times 1.88 \mathrm{~m}$ Industrial Acoustics sound chamber. The video terminal was mounted at eye level on a table within the chamber. Ambient illumination inside the chamber was $1.29 \mathrm{~cd} / \mathrm{m}^{2}$. It was provided by a $40-\mathrm{W}$ bulb housed in a parabolic reflector positioned to diffuse the light evenly, minimizing glare on the screen. Fresh air was provided by a fan mounted in the ceiling of the chamber. An Apple Ile microcomputer orchestrated the presentation of critical signals and neutral events in all experimental conditions. Responses occurring within $1.5 \mathrm{sec}$ after the onset of a critical signal were recorded by the computer as correct detections. All other responses were recorded as errors of commission, or false alarms. The 1.5-sec cutoff was selected on the basis of previous work with this task, which indicated that if subjects were to respond to a stimulus event they would do so within that interval (Galinsky, Warm, Dember, Weiler, \& Scerbo, 1990). Subjects surrendered their watches upon reporting to the laboratory and had no knowledge of the length of the vigil other than it would not exceed $2 \mathrm{~h}$. Computer control of all aspects of the study afforded an opportunity to keep experimenter/subject contact during the vigil to a minimum.

\section{RESULTS}

Preliminary inspection of the data indicated a genderrelated difference in the choice of hard and easy conditions. Of the 20 women who were offered a choice, 18 selected the easy task, whereas the men split 10 and 10 $\left[\chi^{2}(1\right.$, corrected for continuity $\left.)=5.83, p<.025\right]$. As a result of this finding, gender was included as an experimental factor in all subsequent analyses of the data.

Percentages of correct detections and false alarms were determined for each subject in all experimental conditions. Detection probability scores were converted to arcsines and tested for statistical significance in a 2 (choice) $\times$ 2 (signal salience) $\times 2$ (gender) $\times 5$ (periods of watch) analysis of variance. The results of that analysis revealed no significant main effects for choice, salience, or gender ( $p>.05$ in each case). However, detection probability was found to decline significantly over time $[F(4,288)=$ $58, p<.001]$, and there were significant interactions between periods and choice $[F(4,288)=2.77, p<.027]$ and salience and gender $[F(1,72)=4.32, p<.039]$.

The periods $\times$ choice interaction is displayed in Figure 1 , wherein mean detection probability scores are plotted as a function of time on watch for the choice and no-choice conditions. It is evident in the figure that while performance efficiency declined over time in both conditions, the magnitude of the vigilance decrement was considerably less among subjects in the choice condition.

With regard to the salience $\times$ gender interaction, tests of the simple effects of salience within gender indicated that male subjects detected significantly more high-salience $(M=70 \%)$ than low-salience signals $(M=57 \%)[F(1,72)$ $=5.49, p<.025]$. In contrast, signal salience had an inconsequential effect upon the frequency of signal detections

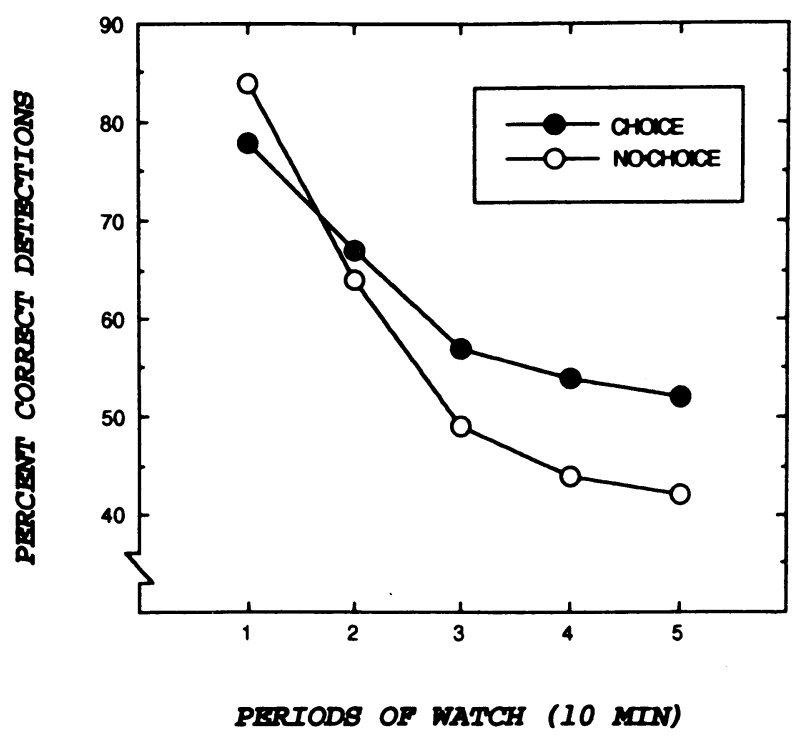

Figure 1. Percentage of correct detections as a function of periods of watch for the choice and no-choice conditions. 
among female subjects ( $M$ s for the high- and low-salience conditions $=53 \%$ and $57 \%$, respectively, $F<1$ ).

False alarm percentages were also converted to arcsines and submitted to a similar analysis of variance as the detection probability scores. The results of that analysis indicated that false alarms were significantly more prevalent in the low-salience $(M=2.49 \%)$ than in the high-salience $(M=1.24 \%)$ condition $[F(1,72)=5.77, p<.018]$ and that frequency of false alarms declined with time on watch ( $M$ s for Periods $1-5=3.18 \%, 1.69 \%, 1.52 \%, 1.49 \%$, and $1.45 \%$, respectively) $[F(4,288)=7.6, p<.001]$. All of the remaining sources of variance in the analysis lacked significance ( $p>.05$ in each case).

\section{DISCUSSION}

The present study was designed to test the hypothesis that affording subjects an opportunity to select the condition in which they were to participate would increase their task commitment and attenuate the performance decrement that is typically found in vigilance experiments. The results clearly supported that hypothesis. The vigilance decrement was indeed attenuated in the choice condition as opposed to the nonchoice condition. In this regard, it is of interest that a significant choice $X$ time interaction was found only in connection with the frequency of signal detections; there were no significant effects associated with choice in the false alarm scores. From a signal detection theory perspective (Davies \& Parasuraman, 1982), a result of this sort implies that the stabilizing effects of the choice variable were perceptual in origin and not simply the result of an increased willingness on the part of the choice subjects to emit detection responses. It is also of interest that the beneficial effect of choice on performance stability was quite robust. It occurred even though the opportunity for choice was illusory, since subjects were in reality assigned to task conditions at random. Moreover, the absence of any significant interactions between choice and gender or choice and signal salience indicated that the effect of choice appeared equally in the data of the male and female subjects and that it was independent of the degree of signal salience.

The independence of choice and salience may, at first glance, seem to contradict Brickman's (1987) suggestion that the effects of choice would be more evident in a difficult as opposed to an easy task. However, the conditions of this study may not have provided a proper forum for testing that aspect of Brickman's view. Although the duration disparity that differentiated critical signals from neutral events was greater in the high- as opposed to the low-salience condition, there is reason to believe that the former condition was not truly easy in an absolute sense. Using a display identical to the high-salience condition of the present study, Galinsky (1991) has reported that this condition is associated with several symptoms of fatigue, such as eye strain, neckache, backache, and so forth, as a result of the need to continuously monitor the video terminal and to remain in a constrained posture while doing so. Hence, it is possible that the signal salience conditions employed in the present study both posed rather difficult tasks and that they were not sufficiently different to furnish an adequate test of the notion that the beneficial effects of choice would be maximized under the conditions of a difficult task.

The results of this study provide additional support for the relation between motivation and performance in sustained attention tasks and for Smith's (1966) claim that motivational factors play an important role in the vigilance decrement. It is worth noting that the motivational techniques described earlier that have been used to enhance the quality of vigilant behavior, such as nonveridical knowledge of results about performance efficiency, financial and course incentives, and subject surveillance, focus on extrinsic sources of motivation-that is, sources that are independent of the task itself and that are controlled by someone other than the subject (Eysenck, 1982). However, as Deci (1975) has noted, intrinsic sources of motivation, those inherent in the task itself, can contribute to performance efficiency in a variety of situations, and this is also the case with regard to vigilance. Dember and his co-workers have shown that vigilance tasks that pose a cognitive challenge and are thereby more interesting than the typical vigilance task are characterized by stable, or even improved, performance over the course of the vigil (Dember, Warm, Bowers, \& Lanzetta, 1984). The present findings confirm and extend the results of Dember et al. by showing that intrinsic factors arising from subjects' having a sense of choice can also moderate the vigilance decrement.

The predicted effects of choice in this study were accompanied by two unanticipated results, both involving gender. One of these was the finding that male subjects were evenly divided in their selection of the hard and easy task options, while women subjects selected the easy option by a substantial margin. Although we cannot be certain of the determining factors in this gender-related effect, we can point to data indicating that women tend to be more concerned with work achievement than men (see Halpern, 1986) and that women often have a lower expectancy of success than men (see Weiner, 1985). Hence, it is conceivable that factors such as these led women in this study to bias their choice toward the condition that would provide the greater opportunity for task accomplishment.

The second unanticipated outcome was the interaction between gender and signal salience. The fact that the performance of the males improved in the high-salience as opposed to the low-salience condition is not surprising, since other studies have also demonstrated a positive relation between salience and performance efficiency in sustained attention tasks (Warm \& Jerison, 1984). What is surprising is the finding that female subjects did not profit from increased signal salience. Previous studies have generally reported sex differences to be negligible in vigilance tasks (Berch \& Kanter, 1984); when such differences do appear, they are likely to be associated with spatial but not temporal discriminations, such as those required herein (Dittmar, 1989). Clearly, the unusual gender $x$ salience interaction merits further study.

\section{REFERENCES}

BerCh, D. B., KANTER, D. R. (1984). Individual differences. In J. S. Warm (Ed.), Sustained attention in human performance (pp. 143-178). Chichester, U.K.: Wiley.

BeVAN, W., \& TURNER, E. D. (1965). Vigilance performance with a qualitative shift in reinforcers. Journal of Experimental Psychology, 70, 83-86.

Brehm, J. W., Cohen, A. R. (1962). Explorations in cognitive dissonance. New York: Wiley.

Brickman, P. (1987). Commitment, conflict, and caring. Englewood Cliffs, NJ: Prentice-Hall.

Davies, D. R., \& Parasuraman, R. (1982). The psychology of vigilance. London: Academic Press.

DECI, E. L. (1975). Intrinsic motivation. London: Plenum.

Dember, W. N., WArm, J. S., Bowers, J. C., \& LANZetTa, T. (1984). Intrinsic motivation and the vigilance decrement. In A. Mital (Ed.), Trends in ergonomics/Human factors I (pp. 21-26). Amsterdam: Elsevier.

DITTMAR, M. L. (1989). Sex differences and stress in vigilance performance. Unpublished doctoral dissertation, University of Cincinnati, Cincinnati, $\mathrm{OH}$.

EYSENCK, M. (1982). Attention and arousal: Cognition and performance. New York: Springer-Verlag.

Galinsky, T. L. (1991). Psychophysical determinants of stress in sustained attention. Unpublished doctoral dissertation, University of Cincinnati, Cincinnati, $\mathrm{OH}$.

Galinsky, T. L., Warm, J. S., Dember, W. N., Weiler, E. M., \& SCERBO, M. W. (1990). Sensory alternation and vigilance performance: The role of pathway inhibition. Human Factors, 32, 717-728.

HaLCOMB, C. G., BLACKWELL, P. (1969). Motivation and the human monitor: I. The effect of contingent credit. Perceptual \& Motor Skills, 28, 623-629.

HALPERN, D. F. (1986). Sex differences in cognitive abilities. Hillsdale, NJ: Erlbaum.

Hovanitz, C. A., Chin, K., WARM, J. S. (1989). Complexities in life stress-dysfunction relationships: A case in point-tension headache. Journal of Behavioral Medicine, 12, 55-75.

LOEB, M., SCHMidT, E. A. (1963). A comparison of the effects of different kinds of information in maintaining efficiency on an auditory monitoring task. Ergonomics, 6, 75-81. 
NACHREINER, F. (1977). Experiments on the validity of vigilance experiments. In R. R. Mackie (Ed.), Vigilance: Theory, operational performance and physiological correlates (pp. 665-678). New York: Plenum. Putz, V. R. (1975). The effects of different modes of supervision on vigilance behaviour. British Journal of Psychology, 66, 157-160.

RODIN, J., \& LANGER, E. (1977). Long-term effects of a control-relevant intervention among the institutionalized aged. Journal of Personality \& Social Psychology, 35, 897-902.

Sirowicz, R. R., WARE, J. R., \& BAKER, R. A. (1962). The effects of reward and knowledge of results on the performance of a simple vigilance task. Journal of Experimental Psychology, 64, 58-61.

SMith, R. L. (1966). Monotony and motivation: A theory of vigilance. Santa Monica, CA: Dunlap and Associates.

WARM, J. S. (1984). An introduction to vigilance. In J. S. Warm (Ed.), Sustained attention in human performance (pp. 1-14). Chichester, U.K.: Wiley.
Warm, J. S., Dember, W. N., \& Parasuraman, R. (1991). Effects of olfactory stimulation on performance and stress in a visual sustained attention task. Journal of the Society of Cosmetic Chemists, 42, 199-210.

WARM, J. S., EpPS, B. D., \& Ferguson, R. P. (1974). Effects of knowledge of results and signal regularity on vigilance performance. Bulletin of the Psychonomic Society, 4, 272-274.

WARM, J. S., \&ERISON, H. J. (1984). The psychophysics of vigilance. In J. S. Warm (Ed.), Sustained attention in human performance (pp. 15-59). Chichester, U.K.: Wiley.

WEINER, B. (1985). Human motivation. New York: Springer-Verlag.

(Manuscript received November 19, 1991.) 